\title{
Peso corporal y depresión en adultos mayores de comunidad urbano marginal en México - Tamaulipas.
}

\author{
Duran-Badillo Tirso ${ }^{1, a}$, Martínez Aguilar Ma. de la Luz ${ }^{2, b}$, Gutiérrez Sánchez Gustavo ${ }^{2, c}$, \\ Aguilar Hernández Rosa Ma. 2,d, Guevara-Valtier Milton Carlos ${ }^{2, e}$
}

\begin{abstract}
RESUMEN
El peso corporal inadecuado y la depresión son factores que alteran la calidad de vida del adulto mayor. Objetivos: identificar asociación existente entre peso corporal y depresión en el adulto mayor. Material y Metodos: estudio descriptivo y correlacional, se realizó en una población urbano-marginal de Tamaulipas, México. Participaron 252 adultos mayores de 60 años y que tuvieran la capacidad física para mantenerse de pié al momento de medir el peso corporal y talla. El muestreo fue no probabilístico, por conveniencia. Para evaluar el peso corporal, se calculó Índice de Masa Corporal (IMC) y para conocer el estado depresivo, se utilizó la Escala de Depresión Geriátrica en su versión completa (GDS-30) con una sensibilidad del 84\% y especificidad del 95\%. Procesamiento y análisis de datos en SPSS mediante correlación de Spearman. Resultados: bajo peso $(1,6 \%)$, peso normal $(61,5 \%)$, sobre peso $(32,9 \%)$ y obesidad (4,0\%). Sin depresión $40,1 \%$, depresión leve $34,1 \%$ y depresión establecida $25,0 \%$. Correlación entre peso corporal y depresión $\left(\mathrm{r}_{\mathrm{s}}=0,246 ; p<0,001\right)$. Discusión: la correlación encontrada es similar a lo reportado en otro estudio. Conclusiones: escasa asociación entre ambas variables; casi la mitad de los adultos mayores estudiados tenían un problema de malnutrición y más de la mitad depresión.
\end{abstract}

PALABRAS CLAVE: Peso corporal, depresión, anciano, índice de masa corporal.

\section{Body weight and depression in elderly marginal urban community in Mexico - Tamaulipas.}

\begin{abstract}
SUMMARY
Improper body weight and depression are factors that affect the quality of life of the older people. Objectives: identify association between body weight and depression in the older people. Material and Methods: descriptive and correlational study was conducted in a marginal urban population from Tamaulipas, Mexico. Participants 252 adults aged 60 years and had the physical ability to keep standing when measuring body weight and height. The sampling was not probabilistic, convenience. To assess body weight, body mass index was calculated and for the depressive state, the Geriatric Depression Scale was used in its full version (GDS-30) with a sensitivity of $84 \%$ and specificity of 95\%. Processing and data analysis in SPSS using Spearman correlation. Results: underweight (1.6\%), normal weight (61.5\%), overweight (32.9\%) and obesity (4.0\%). No depression 40.1\%, 34.1\% mild depression and $25.0 \%$ established depression. Correlation between body weight and depression $\left(r_{\mathrm{s}}=0.246, p<0.001\right)$. Discussion: the correlation found is similar to that reported in another study. Conclusions: little association between the two variables. Almost half of older people surveyed had malnutrition problem and over half depression.
\end{abstract}

KEYWORDS: Body weight, depression, aged, body mass index.

Unidad Académica Multidisciplinaria Matamoros, Universidad Autónoma de Tamaulipas. Matamoros, Tamaulipas, México.

Facultad de Enfermería, Universidad Autónoma de Nuevo León. Nuevo León, México.

Máster en Gerontología Social ; ${ }^{\mathrm{b}}$ Dra. en Enfermería y Cultura de los Cuidados ; Doctor en Psicología Internacional ;

Dra. en Educación Internacional ; ${ }^{e}$ Dr. en Ciencias de la Educación 


\section{INTRODUCCIÓN}

Existen diferentes factores y situaciones de la vida que afectan la autonomía del adulto mayor (1), entre las que se encuentra el estado de nutrición y depresión. El peso corporal es el principal indicador que orienta la condición nutricional en la que se encuentra el adulto mayor. La OMS sugiere la medición a través del Índice de Masa Corporal (IMC) y lo clasifica en bajo peso, normopeso, sobre peso y obesidad $(2,3)$. En México se han reportado prevalencias de bajo peso de $1,8 \%$, sobrepeso $38,9 \%$ y obesidad $35,5 \%$ en adultos mayores (4).

En los adultos mayores el peso está influenciado por cambios biológicos y fisiológicos propios del envejecimiento, como la disminución del metabolismo, pérdida de tejido magro, disminución del tono y masa muscular, absorción de agua en el tejido celular, y cambios en el tejido óseo (5); así como la pérdida de piezas dentales, caries y problemas de las encías (6). Éstos últimos provocan que el adulto mayor se alimente no con lo que necesita, si no con lo que puede, de fácil ingesta y alto valor calórico.

Se ha reportado que los adultos mayores tienden a presentar un bajo nivel de actividad, lo cual, aunado a malos hábitos alimenticios constituyen un factor de riesgo para la nutrición inadecuada que conlleva a sobrepeso u obesidad (7). El sobrepeso se asocia con una mayor prevalencia de factores de riesgo cardiovascular es como hipertensión, dislipidemias, diabetes mellitus, cardiopatía coronaria y osteoartritis (2).

En lo que respecta a la depresión en los adultos mayores, se encontraron prevalencias que oscilan entre $29 \%$ y el $66,0 \%$, esto en estudios desarrollados en población mexicana (8-10). La evidencia científica señala que es un serio problema en los adultos mayores, debido a que es la etapa de vida en la que se presentan más pérdidas entre las que destacan, la de familiares y amigos, deterioro de la salud física y cognitiva y abandono familiar. Así mismo, la depresión en el adulto mayor conlleva consecuencias que van desde alteración del bienestar hasta el suicidio (11).

Se piensa que el estado del peso corporal del adulto mayor puede estar relacionado con la depresión ya que la última, es una alteración del estado de ánimo que genera pérdida de interés para la ejecución de las actividades de vida incluyendo la de alimentación. El estado emocional afecta la ingesta de alimentos, la literatura indica que algunas personas usan el alimento como una fuente de confort y seguridad, y el exceso de comer puede conducir a la obesidad (12). Por el contrario, la depresión causa disminución del apetito que conduce a la pérdida de peso (13). Esta situación resulta preocupante porque se sabe que el déficit nutricional y la depresión conllevan a la dependencia $(2,8)$ y sus complicaciones van desde el desarrollo y/o exacerbación de enfermedades crónico degenerativas, hasta hospitalizaciones prolongadas.

El fenómeno del peso corporal en relación con la depresión en el adulto mayor es un tema poco abordado, el único estudio que se identificó es el desarrollado por Centurión y col., en población española, en el que reportaron asociación entre peso corporal y depresión de 0,261 (14).

$\mathrm{Al}$ conocer que la depresión puede ser causa-efecto del peso corporal y las repercusiones en la salud del adulto mayor que ambas conllevan, se realizó éste estudio con el objetivo de identificar asociación existente entre peso corporal y depresión en el adulto mayor, para aportar conocimiento científico que oriente al equipo multidisciplinario de salud en la toma de decisiones y creación de medidas preventivas orientadas a la búsqueda de calidad de vida del adulto mayor, donde el profesional de Enfermería juega un papel importante en la reorientación de hábitos y conductas, a través de la educación y fomento del autocuidado.

\section{MATERIAL Y METODOS}

Estudio descriptivo correlacional, la población estuvo conformada por adultos mayores de una población urbano-marginal que es área de influencia de una institución del sector salud, en primer nivel de atención. El muestreo fue no probabilístico, por conveniencia(15) debido a que no se cuenta con un marco muestral que permita realizarlo en forma aleatoria. La muestra la conformaron 252 adultos mayores de ambos sexos.

Participaron en el estudio los adultos mayores que eran residentes del área de influencia de la institución de salud, mayores de 60 años y que tuvieran la capacidad física para mantenerse de pié al momento de medir el peso corporal y talla. La antropometría proporciona algunos de los indicadores más importantes usados en la vigilancia de la nutrición, por lo que se hizo medición de talla y peso corporal, con los cuales se calculó el índice de masa corporal $\left(\mathrm{IMC}=\mathrm{kg} / \mathrm{m}^{2}\right)$. El IMC se clasificó de acuerdo con los criterios de corte que propone la OMS para adultos: bajo peso (IMC $<18,5$ ), normopeso (IMC, 18,5 a 24,9), so- 
brepeso (IMC, 25,0 a 29,9) y obesidad (IMC>30,0) $(2,3)$. Se utilizó el Test de Yesavage, el cual es una escala de 30 reactivos para identificar la prevalencia de depresión en adultos mayores. Se reporta para esta escala una sensibilidad del $84 \%$ y una especificidad del $95 \%{ }^{16}$. Es un test de preguntas cerradas con opción de respuesta SI y No, en las preguntas: $1,5,7,9,15,19,21,27,29$ y 30 si la respuesta del sujeto es $\mathrm{NO}$ equivale a un punto, cuando contesta SI corresponde a cero puntos y en el resto de las preguntas es viceversa, al contestar todas las preguntas se suman los puntos obtenidos y el diagnóstico se obtiene con la siguiente tabla: de 0 a 10 puntos es normal, de 11 a 15 tiene depresión leve y más de 15 tiene depresión establecida (17).
La recolección de la información se realizó en visitas domiciliarias por parejas de entrevistadores. Los adultos mayores, previamente identificados a partir del censo del Centro Comunitario, fueron invitados a participar en el estudio; una vez que cumplieron los criterios de inclusión, aceptaron participar en el estudio y firmaron el consentimiento informado, se les aplicó el instrumento de evaluación, para lo que se solicitó un espacio privado y libre de distracciones.

Los datos se capturaron y analizaron en el paquete estadístico IBM SPSS versión 18 para Windows. En el análisis se utilizó estadística descriptiva para caracterización de la muestra, tablas de contingencia y coeficiente de correlación no paramétrica de Spearman. Se siguieron las

Tabla 1. Distribución del peso corporal según variables sociodemográficas y de salud.

\begin{tabular}{|c|c|c|c|c|c|c|c|c|}
\hline \multirow[t]{2}{*}{ IMC } & \multicolumn{2}{|c|}{ Bajo peso } & \multicolumn{2}{|c|}{ Normal } & \multicolumn{2}{|c|}{ Sobre peso } & \multicolumn{2}{|c|}{ Obesidad } \\
\hline & $\mathbf{F}$ & $\%$ & $\mathbf{F}$ & $\%$ & $\mathbf{F}$ & $\%$ & $\mathbf{F}$ & $\%$ \\
\hline \multicolumn{9}{|l|}{ Edad } \\
\hline $60-70$ & 2 & 1,2 & 100 & 61,7 & 55 & 34,0 & 5 & 3,1 \\
\hline 71 y más & 2 & 2,2 & 55 & 61,1 & 28 & 31,1 & 5 & 5,6 \\
\hline \multicolumn{9}{|l|}{ Sexo } \\
\hline Femenino & 3 & 2,0 & 100 & 65,8 & 43 & 28,3 & 6 & 3,9 \\
\hline Masculino & 1 & 1,0 & 55 & 55,0 & 40 & 40,0 & 4 & 4,0 \\
\hline \multicolumn{9}{|l|}{ Escolaridad } \\
\hline Con escolaridad & 1 & 1,6 & 42 & 67,8 & 18 & 29,0 & 1 & 1,6 \\
\hline Sin escolaridad & 3 & 1,6 & 113 & 59,5 & 65 & 34,2 & 9 & 4,7 \\
\hline \multicolumn{9}{|l|}{ Estado Marital } \\
\hline Con pareja & 3 & 2,2 & 84 & 61,8 & 44 & 32,3 & 5 & 3,7 \\
\hline Sin pareja & 1 & 0,9 & 71 & 61,2 & 39 & 33,6 & 5 & 4,3 \\
\hline \multicolumn{9}{|l|}{ Empleo remunerado } \\
\hline $\mathrm{Si}$ & 1 & 4,0 & 13 & 52,0 & 10 & 40 & 1 & 4,0 \\
\hline No & 3 & 1,3 & 142 & 62,6 & 73 & 32,1 & 9 & 4,0 \\
\hline \multicolumn{9}{|c|}{ Realiza actividades domesticas } \\
\hline $\mathrm{Si}$ & 4 & 2,0 & 124 & 62,0 & 63 & 31,5 & 9 & 4,5 \\
\hline No & 0 & 0,0 & 31 & 59,6 & 20 & 38,5 & 1 & 1,9 \\
\hline \multicolumn{9}{|c|}{ Número de padecimientos } \\
\hline Ninguno & 0 & 0,0 & 46 & 59,7 & 24 & 31,2 & 7 & 9,1 \\
\hline Entre uno y dos & 4 & 2,5 & 94 & 60,3 & 56 & 35,9 & 2 & 1,3 \\
\hline Tres y más & 0 & 0,0 & 15 & 78,9 & 3 & 15,8 & 1 & 5,3 \\
\hline \multicolumn{9}{|c|}{ Número de medicamentos } \\
\hline Entre cero y cuatro & 4 & 1,7 & 137 & 59,6 & 80 & 34,8 & 9 & 3,9 \\
\hline Cinco y más & 0 & 0,0 & 18 & 81,8 & 3 & 13,6 & 1 & 4,6 \\
\hline
\end{tabular}

Fuente: Índice de Masa Corporal, Cedula de Datos Personales 
Tabla 2. Distribución de depresión según variables sociodemográficas y de salud.

\begin{tabular}{|c|c|c|c|c|c|c|}
\hline \multirow[t]{2}{*}{ Depresión } & \multicolumn{2}{|c|}{ Normal } & \multicolumn{2}{|c|}{ Leve } & \multicolumn{2}{|c|}{ Establecida } \\
\hline & $\mathbf{F}$ & $\%$ & $\mathbf{F}$ & $\%$ & $\mathbf{F}$ & $\%$ \\
\hline \multicolumn{7}{|l|}{ Edad } \\
\hline $60-70$ & 66 & 40,7 & 54 & 33,3 & 42 & 26,0 \\
\hline 71 y más & 35 & 38,9 & 34 & 37,8 & 21 & 23,3 \\
\hline \multicolumn{7}{|l|}{ Sexo } \\
\hline Femenino & 63 & 41,4 & 54 & 35,6 & 35 & 23,0 \\
\hline Masculino & 38 & 38,0 & 34 & 34,0 & 28 & 28,0 \\
\hline \multicolumn{7}{|l|}{ Escolaridad } \\
\hline Con escolaridad & 78 & 41,1 & 66 & 34,7 & 46 & 24,2 \\
\hline Sin escolaridad & 23 & 37,1 & 22 & 35,5 & 17 & 27,4 \\
\hline \multicolumn{7}{|l|}{ Estado Marital } \\
\hline Con pareja & 58 & 42,6 & 44 & 32,4 & 34 & 25,0 \\
\hline Sin pareja & 43 & 37,1 & 44 & 37,9 & 29 & 25,0 \\
\hline \multicolumn{7}{|l|}{ Empleo remunerado } \\
\hline $\mathrm{Si}$ & 9 & 36,0 & 9 & 36,0 & 7 & 28,0 \\
\hline No & 92 & 40,5 & 79 & 34,8 & 56 & 24,7 \\
\hline \multicolumn{7}{|c|}{ Realiza actividades domesticas } \\
\hline $\mathrm{Si}$ & 85 & 42,5 & 72 & 36,0 & 43 & 21,5 \\
\hline No & 16 & 30,8 & 16 & 30,8 & 20 & 38,4 \\
\hline \multicolumn{7}{|c|}{ Número de padecimientos } \\
\hline Ninguno & 23 & 29,8 & 29 & 37,7 & 25 & 32,5 \\
\hline Entre uno y dos & 67 & 42,9 & 55 & 35,3 & 34 & 21,8 \\
\hline Tres y más & 11 & 57,8 & 4 & 21,1 & 4 & 21,1 \\
\hline \multicolumn{7}{|c|}{ Número de medicamentos } \\
\hline Entre cero y cuatro & 90 & 39,1 & 82 & 35,7 & 58 & 25,2 \\
\hline Cinco y más & 11 & 50,0 & 6 & 27,3 & 5 & 22,7 \\
\hline
\end{tabular}

Fuente: Test de Yesavage-30, Cédula de datos personales.

Tabla 3. Asociación entre el peso corporal y depresión.

\begin{tabular}{lcccc}
\hline & \multicolumn{2}{c}{ Peso corporal } & \multicolumn{2}{c}{ Depresión } \\
& $\mathbf{r}_{\mathbf{s}}$ & $\mathbf{p}$ & $\mathbf{r}_{\mathbf{s}}$ & $\mathbf{p}$ \\
\hline Número de medicamentos que consume & $-0,128$ & 0,043 & & \\
Número padecimientos & $-0,124$ & 0,049 & $-0,239$ & $<0,001$ \\
Realización de actividades domésticas & & & 0,142 & 0,024 \\
Peso corporal & & & 0,246 & $<0,001$ \\
\hline
\end{tabular}

consideraciones éticas contenidas en los artículos 21 y 22 del Reglamento de la Ley General de Salud en materia de investigación para la Salud de la Secretaría de Salud (18), en cuanto a respetar el anonimato, mantener la confidencialidad y dar a conocer verbalmente y por escrito los posibles riesgos por participar en el estudio.
El estudio se consideró de bajo riesgo, por lo que en la medición de peso y talla uno de los entrevistadores permaneció al lado del adulto mayor para minimizar el riesgo de caídas, se presentó por escrito el consentimiento informado y se recabó la firma de los adultos mayores. Así mismo se contó con la aprobación del comité de Ética e 
investigación de la Unidad Académica Multidisciplinaria Matamoros de la Universidad Autónoma de Tamaulipas.

\section{RESULTADOS}

Participaron en el estudio 252 adultos mayores, de ellos el $60,3 \%$ eran del sexo femenino, la mayoría tenía entre 60 y 70 años $(64,2 \%)$, más de la mitad con esposo/a o concubino/a (54,0\%); el 75,4\% con escolaridad, sin empleo remunerado $(90,1 \%)$, el $79,4 \%$ realizaba actividades domésticas, el 91,2\% consumía entre cero y cuatro medicamentos y el $69,5 \%$ tenía entre uno y cuatro padecimientos.

El peso corporal se distribuyó de la siguiente manera: $1,6 \%$ de los adultos mayores tenía bajo peso, $61,5 \%$ peso normal, 32,9\% sobre peso y el 4,0\% obesidad. Se identificó mayor prevalencia de sobrepeso en el rango de edad de 60 a 70 años (34,0\%), en el sexo masculino (40,0\%), los que no tenían escolaridad $(34,2 \%)$ y los que no realizaban actividades domésticas $(38,5 \%)$ (Tabla 1$)$.

El 40,1\% (101 personas) no tenía depresión, seguida del $34,9 \%$ (88 personas) con depresión leve y el $25 \%$ (63 personas) depresión establecida. Resultó predominante la depresión leve en el grupo de 71 años y más $(37,8 \%)$ y establecida en el grupo de 60 a 70 años $(26,0 \%)$. La depresión entre leve y establecida fue mayor en los adultos mayores del sexo masculino $(62,0 \%)$, en quienes no tienen escolaridad $(62,9 \%)$ y que no tienen pareja $(62,9$ $\%$ ) (Tabla 2).

En la tabla 3, se puede apreciar los resultados obtenidos mediante el análisis bivariado con el coeficiente de correlación de spearman $\left(\mathrm{r}_{\mathrm{s}}\right)$ entre las variables de peso corporal y depresión con otras variables analizadas, donde se puede observar que solamente se encontró el $24,6 \%$ de asociación entre el peso corporal y la depresión.

\section{DISCUSIÓN}

Las alteraciones del peso corporal y presencia de depresión, son situaciones que afectan el bienestar del adulto mayor, la dinámica familiar y generan gastos económicos para el sector salud y familia. Dichos gastos derivan de las complicaciones relacionadas con estos problemas y en muchos casos, necesidad de hospitalización. Aunque la literatura sugiere posible asociación entre ambas variables $(12,13)$, los resultados del presente estudio no mostraron la asociación esperada. El resultado encontrado se acerca a lo reportado por Centurión y cols. (14), quienes reportaron resultados de las mismas variables de 0,261.
De acuerdo con las consecuencias que el peso corporal inadecuado con lleva ${ }^{2}$ resulta preocupante lo encontrado en el presente estudio, donde se identificó que casi un tercio de la muestra estudiada presentó algún grado de alteración del peso, lo que sugiere la necesidad de diseñar estrategias de intervención para disminuir los riesgos relacionados con el bajo peso, sobrepeso y obesidad.

Los resultados de este estudio en cuanto a sobrepeso son similares a lo encontrado por Hernández y Linares en un estudio realizado con adultos mayores de una comunidad de Cuba (19); sin embargo discrepan con lo que reporta Shama-Levy, Cuevas-Nasu, Mundo-Rosas, Morales-Ruán, Cervantes-Turrubiates y Villalpando-Hernández (20), quienes reporta hallazgos superiores a los del presente. Las diferencias entre ambos estudios se pudieran justificar en que éste estudio se desarrolló con una muestra compuesta por adultos mayores de una comunidad urbana-marginal y el reporte de Shama-Levy y cols., es resultado de una encuesta nacional (20).

En cuanto a la frecuencia de obesidad encontrada en el presente estudio el resultado es menor a lo reportado por otros autores (20-23), dichas diferencias pudieran derivar del contexto de estudio; ya que este estudio fue desarrollado en comunidad y los de comparación se implementaron en estancias hospitalarias, consulta médica y de enfermería.

En cuanto al estado de depresión se identificó que casi dos tercios de la muestra estudiada presentaron algún grado de depresión, dicho hallazgo se acerca a lo reportado por Calderón, y cols.(21), Contreras, y cols. (24), y Pérez, y cols (25). No obstante, es mayor a lo que indica Cortés, y cols. (23), Gonzáles, y cols. (22), Estrada, y cols. (26) y Borda y cols. (27). En cuanto a las características de los adultos mayores con depresión se encontró que es más frecuente en el sexo femenino y en los adultos de mayor edad, similar a lo reportado por Alves y Soares (28) y Estrada y col. (26).

\section{CONCLUSIONES}

Los resultados de este estudio muestran que casi la mitad de los adultos mayores estudiados tenían malnutrición y más de la mitad depresión. La asociación encontrada entre ambas variables fue escasa. Se reconoce que haber utilizado un muestreo por conveniencia es una limitante para la generalización de los resultados, sin embargo, lo encontrado con este estudio es un aporte importante para la disciplina de enfermería, ya que es un acercamiento y orientación para la creación de medidas preventivas 
orientadas al cuidado de la salud nutricional y emocional del adulto mayor.

\section{REFERENCIAS BIBLIOGRAFICAS}

1. Giraldo CI, Franco GM. Capacidad funcional y salud: orientaciones para cuidar al adulto mayor. Av Enferm. 2008; 26(1):43-58.

2. Organización Mundial de la Salud. El estado físico: uso e interpretación de la antropometría. Informe de un comité de expertos de la OMS. Ginebra: Organización Mundial de la Salud; 1995.

3. Organización Mundial de la Salud. Obesidad y sobrepeso. Nota descriptiva No. 311. Ginebra: Organización Mundial de la Salud; 2012.

4. Shamah-Levy T, Cuevas-Nasu L, Mundo-Rosas V, Morales-Ruán C, Cervantes-Turrubiates L, VillalpandoHernández S. Estado de salud y nutrición de los adultos mayores en México: resultados de una encuesta probabilística nacional. Salud Pública Mex. 2008; 50:383-389.

5. Hayflick, L. Cómo y por qué envejecemos. España: Herder; 1999.

6. De Nicola P. Geriatría. México DF: El Manual Moderno; 1985.

7. Reyes-Audiffred V, Sotomayor-Sánchez SM, GonzálezJuárez L. Conductas relacionadas con la salud del adulto mayor en una comunidad suburbana del Distrito Federal. Revista de Enfermería del Instituto Mexicano del Seguro Social. 2007; 15(1):27-31.

8. Ávila-Funes JA, Melano-Carranza E, Payette H, Amieva H. Síntomas depresivos como factor de riesgo de dependencia en adultos mayores. Salud Pública Méx. 2007; 49(5): 367-375.

9. Ávila-Funes JA, Garant ME, Aguilar-Navarro S. Relación entre los factores que determinan los síntomas depresivos y los hábitos alimentarios en adultos mayores de México. Rev. Panam Salud Publica. 2006; 19(5): 321-330.

10.Duran-Badillo T, Aguilar RM, Martínez ML, Gutiérrez G, Vázquez L. Salazar-González BC. Asociación de velocidad de marcha y síntomas depresivos en adultos mayores de una comunidad urbano-marginal. Desarrollo Científico de Enfermería. 2011; 19(2): 4447.

11. Organización Mundial de la Salud. Tratamiento farmacológico de los trastornos mentales en la atención primaria de salud. Washington, D.C.: OPS. 2010. (Citado el 20 noviembre 2011) Disponible en: http://bit.ly/15v1rcW
12.Roper N, Logan W, Tierney AJ. The Roper-LoganTierney: Model of nursing based on activities of living. China: Churchill Livingstone; 2004.

13. Antón M, Gálvez N, Esteban R. Depresión y ansiedad. En: Tratado de Geriatría para residentes. Madrid, España: Sociedad Española de Geriatría y Gerontología; 2007.

14.Centurión L, Aparicio A, Ortega RM, RodríguezRodríguez E, López-Plaza B \& López-Sobaler AM. Asociación entre riesgo de desnutrición y capacidad afectiva en personas de edad avanzada institucionalizadas de la Comunidad de Madrid. Nutrición Clínica y Dietética Hospitalaria. 2010; 30(2): 33-41.

15.Polit DF, Hungler BP. Investigación Científica en Ciencias de la Salud.6 ${ }^{a}$. ed. México: McGraw-Hill Interamericana; 2005.

16. Beamud D, Bosch JM, Castro L, et al. Plan de formación a distancia SCMFIC-SMMFYC. Personas mayores: fisiología del envejecimiento. Unidad didácticas 1. Evaluación del paciente mayor. Barcelona: Doyma, 1998.

17.García MJ, Tobías J. Prevalencia de depresión en mayores de 65 años. Perfil del anciano de riesgo. Atención primaria. 2001; 27(7):484-488.

18. Secretaria de Salud. Reglamento de la Ley General de Salud en materia de investigación para la salud. Mexico DF: Secretaria de Salud; 1987.

19.Hernández Y, Linares EM. Estado nutricional del adulto mayor en un área de salud de la ciudad de Pinar del Río, Cuba. Revista Cubana de Alimentación y Nutrición. 2010; 20(1):57-71.

20.Shamah-Levy T, Cuevas-Nasu L, Mundo-Rosas V, Morales-Ruán C, Cervantes-Turrubiates L, VillalpandoHernández S. Estado de salud y nutrición de los adultos mayores en México: resultados de una encuesta probabilística nacional. Salud Publica Mex. 2008; 50:383-389.

21.Calderón ME, Ibarra F, García J, Gómez C, RodríguezOrozco AR. Evaluación nutricional comparada del adulto mayor de consultas de medicina familiar. Nutrición Hospitalaria. 2010; 25 (4): 669-675.

22. González IAL, Barragán AJ, Valdovinos SB. Pérdida de peso en el adulto mayor durante la hospitalización. Ciencias Clínicas. 2012, 28(9):8-20.

23. Cortés AR, Villarreal E, Galicia L, Martínez L, Vargas ER. Evaluación geriátrica integral del adulto mayor. Revista Médica de Chile. 2011; 139: 725-731.

24.Contreras AL, Mayo GVA, Romaní DA, et al. Malnutrición del adulto mayor y factores asociados en el distrito de Masma Chicche, Junín, Perú. Rev Med Hered. 2013; 24: 186-191. 
25.Pérez E, Lizárraga DC, Martínez MR. Asociación entre desnutrición y depresión en el adulto mayor. Nutrición Hospitalaria. 2014; 29(4): 901-906.

26.Estrada A, Cardona D, Segura AM, Ordóñez J, Osorio JJ. Síntomas depresivos en adultos mayores institucionalizados y factores asociados. Universitas Psychologica. 2013; 12(1): 81-94.

27.Borda M, Anaya MP, Pertuz MM, Romero L, SuárezGarcía A, Suárez A. Depresión en adultos mayores de cuatro hogares geriátricos de Barranquilla (Colombia): prevalencia y factores de riesgo. Salud Uninorte. 2013; 29(1): 64-73.

28. Alves SF, Soares NM. Depressão em idosos institucionalizados no distrito de Bragança. Revista de Enfermagem Referencia. 2011; 3(4): 49-58.

\section{Correspondencia:}

Tirso Duran Badillo

Correo electrónico: tirdb27@hotmail.com

Fecha de recepción: 30 de Octubre 2015

Fecha de aceptación: 19 de Noviembre 2015 